\title{
ANÁLISE DAS ESTRUTURAS DIAMÉTRICA E HIPSOMÉTRICA DE FRAGMENTOS FLORESTAIS LOCALIZADOS NA BACIA DO RIO SÃO FRANCISCO, EM MINAS GERAIS, BRASIL
}

\author{
Lucas Rezende Gomide ${ }^{1}$, José Roberto Soares Scolforo ${ }^{2}$, Antônio Donizette de Oliveira ${ }^{2}$ \\ ${ }^{1}$ Eng. Florestal, M.Sc., Doutorando em Eng. Florestal, UFPR, Curitiba, PR, Brasil - 1rgomide@ hotmail.com \\ ${ }^{2}$ Eng. Florestais, Dr., Depto. de Ciências Florestais, UFLA, Lavras, MG, Brasil - jscolforo@ufla.br - donizete@ufla.br \\ Recebido para publicação: 18/12/2007 - Aceito para publicação: 08/09/2008
}

\begin{abstract}
Resumo
Os objetivos desta pesquisa foram: (a) estabelecer o padrão de distribuição da frequência diamétrica e hipsométrica; (b) realizar inferências sobre a aderência de distribuições (diâmetro e altura), pelo teste de Kolmogorov-Smirnov; (c) verificar a existência de similaridade estrutural entre fragmentos, quando se analisa a distribuição de frequência em diâmetro e altura, na bacia como um todo e dentro de cada grupo fisionômico. A área de estudo compreendeu 20 fragmentos localizados nas áreas ripárias de preservação permanente, distribuídos ao longo da bacia do rio São Francisco, em Minas Gerais. A partir dos resultados encontrados, verificou-se que os grupos mais homogêneos florísticamente apresentaram um maior equilíbrio no padrão da distribuição diamétrica e hipsométrica. Considerando a bacia como um todo e todas as 190 combinações geradas, percebeu-se que $8,95 \%$ das combinações estabeleceram igualdades tanto na distribuição diamétrica como na hipsométrica. Entretanto, apenas 25,26\% estabeleceram igualdade em diâmetro e 12,10\% em altura; a maioria $(53,68 \%)$ não estabeleceu nenhuma igualdade de distribuição nas combinações.

Palavras-chave: Kolmogorov-Smirnov; análise estrutural; distribuição diamétrica e hipsométrica.
\end{abstract}

\begin{abstract}
Diametric and hypsometric structural analysis of native forest fragments localized in the São Francisco river watershed, Minas Gerais, Brazil. The objectives of this research were: (a) to establish the pattern of the diametric and hypsometric frequency distribution; (b) to analyze the distribution adherence (diameter and height), by the Kolmogorov-Smirnov test; (c) to verify the existence of the structural similarity among fragments, when analyze the diameter and height frequency distributions for the watershed as a whole and among physiognomic groups. This study was carried out in 20 native forest fragments localized in riparian permanent preservation areas along the São Francisco river watershed - Minas Gerais. The groups that showed higher floristic homogeneity have more equilibrium for the diametric and hypsometric distribution pattern. Considering the watershed as a whole the 190 combinations formed it was verified that $8,95 \%$ of the combinations were equal for the diametric and hypsometric distributions. Therefore, the same diametric distribution was just $25,26 \%$ and $12,10 \%$ only for hypsometric distribution, and the majority $(53,68 \%)$ were not equal for both distributions.

Keywords: Kolmogorov-Smirnov; structural analysis; diametric and hypsometric distribution.
\end{abstract}

\section{INTRODUÇÃO}

Uma das questões mais importantes na ecologia, de acordo com Pires O’Brien; O’Brien (1995), é compreender por que existe um desequilíbrio na densidade de indivíduos provindos de espécies diferentes na mesma comunidade, uma vez que todos os indivíduos possuem as mesmas chances de se desenvolverem no ambiente. A quantificação desse fenômeno natural, usualmente empregada por pesquisadores, tenta descrever a estrutura da vegetação arbórea por meio de descritores quantitativos como, por exemplo, a densidade, a frequência e a área basal (DISLICH et al., 2001).

Segundo Loetsch et al. (1973), ao se agruparem todos os indivíduos mensurados no inventário florestal, em intervalos definidos de diâmetro, tem-se uma estrutura conhecida como distribuição 
diamétrica da floresta. A estrutura diamétrica de formações florestais inequiâneas torna-se importante, devido à falta da referência de idade dos indivíduos presentes na floresta. Segundo Pulz et al. (1999), o conhecimento da distribuição diamétrica de florestas naturais garante o entendimento de ações/intervenções realizadas no passado, auxiliando ainda na condução do povoamento no futuro. Assim, a produção pode ser planejada a ponto de respeitar a harmonia entre classes de diâmetro, no presente e futuro.

Modelos que utilizam esse tipo de distribuição são empregados na definição de ciclo de corte e de limites de colheita em volume, auxiliam na determinação da viabilidade econômica, produzem inferências sobre as melhores práticas dentro do manejo florestal e contribuem na estruturação do planejamento florestal. Complementando, os estudos fitossociológicos, florísticos e estruturais de remanescentes florestais são importantes também para a adoção de critérios e metodologias visando o manejo, a conservação e a recuperação desses remanescentes (SILVA, 2003). Rodrigues e Gandolfi (1998) afirmam que o conhecimento desses fatores aumenta as chances de recuperação do ambiente.

A distribuição das variáveis dendrométricas (altura e diâmetro, principalmente) na floresta vem sendo analisada por vários autores em inúmeras fisionomias (HUBBELL; FOSTER, 1987; FELFILI; SILVA JÚNIOR, 1993), proporcionando informações a respeito do histórico da floresta, em relação a distúrbios como fogo, pragas e doenças, exploração ou, ainda, crescimento e desenvolvimento.

Diante desses fatos, compreender a estrutura diamétrica e hipsométrica de fragmentos presentes em diferentes fitofisionomias, ao longo dos rios da bacia do rio São Francisco, é uma das etapas cruciais para estabelecer um plano de sua revitalização. Esse fato torna-se mais expressivo quando se trata de uma grande extensão de área de preservação permanente $\left(4.606 \mathrm{~km}^{2}\right)$ e que trabalhos retratando o tema são escassos até o momento. Além do mais, o retorno proporcionado por esses ambientes recuperados é importante, ao exercerem funções reguladoras dos recursos hídricos da bacia, garantirem o equilíbrio à fauna silvestre e regularem o clima local.

Assim, o objetivo proposto neste trabalho é estudar e avaliar o padrão de distribuição da frequência diamétrica e hipsométrica em toda a área da bacia do rio São Francisco, no estado de Minas Gerais, dentro e entre cada grupo fisionômico, além de verificar a existência ou não de similaridades estruturais.

\section{MATERIAL E MÉTODOS}

\section{Descrição da área de estudo}

A área de estudo foi a bacia do Rio São Francisco, no estado de Minas Gerais, compreendida entre a Serra da Canastra e o município de Manga. Ela abrange as regiões do Alto e Médio São Francisco, onde foram amostrados 20 fragmentos florestais, nas áreas de preservação permanente, distribuídos ao longo da bacia (Figura 1).

A caracterização dos fragmentos, indicando a localização e suas descrições ambientais, encontram-se na tabela 1. Assim, as regiões classificadas como Awa apresentam um clima quente e úmido, com chuvas de verão, sendo um clima tropical chuvoso típico, com chuvas concentradas no período de outubro a abril, alcançando mais de $90 \%$ do total anual. O inverno (junho a agosto) é muito seco, com totais mensais inferiores a $20 \mathrm{~mm}$. A temperatura média do mês mais frio (julho) é superior a $18{ }^{\circ} \mathrm{C}$, e antes do período das chuvas, nos meses mais quentes, o mês de setembro apresenta temperaturas geralmente elevadas (NIMER, 1979). A categoria Cwa é de clima mesotérmico, com verões quentes. A média supera os $22{ }^{\circ} \mathrm{C}$. Já a temperatura média dos meses mais frios é inferior a $18{ }^{\circ} \mathrm{C}$. A estação seca é bem definida no período de inverno (EPAMIG, 1978). Regiões classificadas como Bsh constituem um clima tropical semiárido, com verões quentes e secos (SANO et al., 1998). A evaporação é superior à precipitação, sem excedente hídrico, proporcionando seca no verão, quando a temperatura média anual supera $18^{\circ} \mathrm{C}$.

Diante dos inúmeros ambientes característicos de cada comunidade situada ao longo dessa bacia, os 20 fragmentos inventariados foram classificados segundo a classificação de vegetação proposto pelo IBGE (1992). Além disso, os 20 fragmentos foram agrupados em grupos fisionômicos de acordo com a tabela 1, seguindo critérios de análise da vegetação como DCA (Análise de Correlação Retificada), TWINSPAN (Two Way Indicator Species Analysis) e CCA (Análise de Correlação Canônica). 


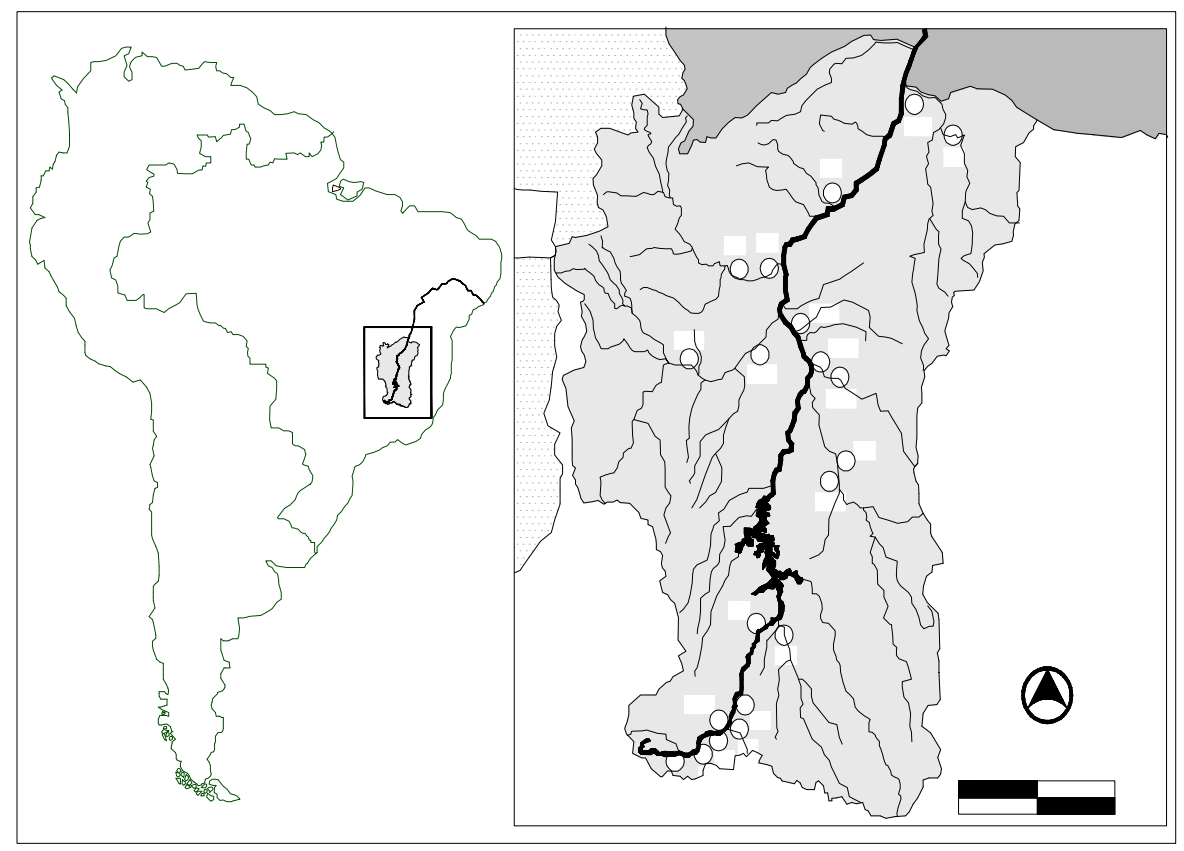

Figura 1. Mapa de localização geral dos 20 fragmentos inventariados, inseridos no complexo da bacia do rio São Francisco, no estado de Minas Gerais, Brasil.

Figure 1. Location map of the 20 inventoried fragments inside the São Francisco river watershed in Minas Gerais state, Brazil.

Tabela 1. Descrição física dos ambientes inventariados, ordenados pela latitude ao longo da bacia do rio São Francisco, no sentido de sua nascente à divisa entre os estados de Minas Gerais e Bahia.

Table 1. Physical Description of the inventoried areas ordered by latitude along the São Francisco river from its spring to the boundary between the state of Minas Gerais and Bahia.

\begin{tabular}{|c|c|c|c|c|c|c|c|c|c|c|c|}
\hline \multirow[b]{2}{*}{ Município } & \multirow[b]{2}{*}{ Frag } & \multicolumn{2}{|c|}{ Coordenadas } & \multirow{2}{*}{$\begin{array}{c}\text { Área } \\
\text { (ha) }\end{array}$} & \multirow{2}{*}{$\begin{array}{l}\text { Altitude } \\
\text { média (m) }\end{array}$} & \multirow[b]{2}{*}{ Clima } & \multirow{2}{*}{$\begin{array}{l}\text { Temperatura } \\
\text { média }\left({ }^{\circ} \mathrm{C}\right)\end{array}$} & \multirow{2}{*}{$\begin{array}{c}\text { Precipitação } \\
\text { anual (mm) }\end{array}$} & \multirow[b]{2}{*}{ Solo predominante * } & \multirow[b]{2}{*}{$\underset{F}{G} \mathbf{V P}$} & \multirow[b]{2}{*}{ AI } \\
\hline & & $\begin{array}{l}\text { Latitude } \\
\text { (S) }\end{array}$ & $\begin{array}{c}\text { Longitude } \\
\text { (W) }\end{array}$ & & & & & & & & \\
\hline Vargem Bonita & 2 & $20^{\circ} 21,06^{\prime}$ & $46^{\circ} 17,47^{\prime}$ & 21,83 & 725,5 & Cwa & 21,8 & 1629,6 & Latossolo vermelho-escuro & I FES & FES \\
\hline Bambuí & 8 & $20^{\circ} 17,01^{\prime}$ & $45^{\circ} 57,54^{\prime}$ & 34,95 & 625,5 & Cwa & 23,7 & 1139,1 & Cambissolo & I FES & FES \\
\hline Iguatama & 12 & $20^{\circ} 09,72^{\prime}$ & $45^{\circ} 46,66^{\prime}$ & 45,12 & 575,5 & Cwa & 21,6 & 1254,6 & Cambissolo & I FES & FES \\
\hline Iguatama & 14 & $20^{\circ} 08,55^{\prime}$ & $45^{\circ} 39,17^{\prime}$ & 43,40 & 575,5 & Cwa & 21,6 & 1254,6 & Latossolo vermelho-escuro & I FES & FES \\
\hline Iguatama $^{\text {a }}$ & 100 & $20^{\circ} 09^{\prime} 21 "$ & $45^{\circ} 50,91^{\prime}$ & 3,78 & 575,5 & Cwa & 21,6 & 1254,6 & Cambissolo & I FES & FES \\
\hline Iguatama $^{\mathrm{a}}$ & 100 & $20^{\circ} 09^{\prime} 26^{\prime \prime}$ & $45^{\circ} 51^{\prime} 7,1^{\prime \prime}$ & 1,39 & 575,5 & Cwa & 21,6 & 1254,6 & Cambissolo & I FES & FES \\
\hline Lagoa da Prata & 17 & $20^{\circ} 00,16^{\prime}$ & $45^{\circ} 37,76^{\prime}$ & 165,26 & 625,5 & Cwa & 22,6 & 1400 & Glei húmico & I FES & FES \\
\hline Martinho Campos & 27 & $19^{\circ} 17,08^{\prime}$ & $45^{\circ} 08,33^{\prime}$ & 15,31 & 525,5 & Cwa & 21 & 1114 & Solos aluviais & I FES & FES \\
\hline Abaeté & 22 & $19^{\circ} 16,07^{\prime}$ & $45^{\circ} 15,82^{\prime}$ & 2,88 & 525,5 & Cwa & 21 & 1377 & Cambissolo & I FES & FES \\
\hline Lassance & 117 & $17^{\circ} 57,01^{\prime}$ & $44^{\circ} 33,14^{\prime}$ & 17,79 & 475,5 & Awa & 22,8 & 1165,5 & Solos aluviais & II FES & $\mathrm{FES}+\mathrm{CSS}$ \\
\hline Lassance & 70 & $17^{\circ} 52,71^{\prime}$ & $44^{\circ} 31,09^{\prime}$ & 41,08 & 475,5 & Awa & 22,8 & 1165,5 & Litossolo & I FES & FES \\
\hline Jequitaí & 116 & $17^{\circ} 09,45^{\prime}$ & $44^{\circ} 37,83^{\prime}$ & 107,64 & 425,5 & Awa & 23,2 & 1031,5 & Litossolo & III FES F & $\mathrm{ES}+\mathrm{FED}+\mathrm{C}$ \\
\hline Brasilândia & 114 & $17^{\circ} 03,67^{\prime}$ & $45^{\circ} 59,02^{\prime}$ & 38,25 & 475,5 & Awa & 22,5 & 1441,5 & Solos aluviais & II FES $\mathrm{F}$ & $\mathrm{FES}+\mathrm{CSS}$ e C \\
\hline Ibiaí & 105 & $17^{\circ} 03,42^{\prime}$ & $44^{\circ} 47,57^{\prime}$ & 15,15 & 425,5 & Awa & 23,7 & 1139,1 & Solos aluviais & III FES F & $\mathrm{ES}+\mathrm{FED}+\mathrm{C}$ \\
\hline Santa Fé de Minas & 112 & $16^{\circ} 54,17^{\prime}$ & $45^{\circ} 19,80^{\prime}$ & 40,00 & 425,5 & Awa & 23,4 & 1153 & Latossolo vermelho-amarelo & II FES $\mathrm{F}$ & $\mathrm{FES}+\mathrm{CSS}$ e C \\
\hline Ibiaí $^{a}$ & 110 & $16^{\circ} 47,47^{\prime}$ & $44^{\circ} 57,56^{\prime}$ & 32,62 & 425,5 & Awa & 23,7 & 1139,1 & Latossolo vermelho-amarelo & III FES F & $\mathrm{ES}+\mathrm{FED}+\mathrm{C}$ \\
\hline Ibiaí $^{\mathrm{a}}$ & 110 & $16^{\circ} 46 ' 55,6^{\prime \prime}$ & $44^{\circ} 58^{\prime} 26,3^{\prime \prime}$ & 14,54 & 425,5 & Awa & 23,7 & 1139,1 & Latossolo vermelho-amarelo & III FES F & $\mathrm{ES}+\mathrm{FED}+\mathrm{C}$ \\
\hline Urucuia & 55 & $16^{\circ} 13,66^{\prime}$ & $45^{\circ} 32,27^{\prime}$ & 9,34 & 425,5 & Awa & 24,3 & 1103 & Podzólico vermelho-amarelo & IV FES & FED \\
\hline São Romão & 66 & $16^{\circ} 12,19^{\prime}$ & $45^{\circ} 10,45^{\prime}$ & 40,57 & 425,5 & Awa & 24 & 1006 & Areia quartzosa & III FES F & $\mathrm{ES}+\mathrm{FED}+\mathrm{C}$ \\
\hline Januária & 77 & $15^{\circ} 35,92^{\prime}$ & $44^{\circ} 42,58^{\prime}$ & 4,74 & 425,5 & Awa & 24,4 & 975,81 & Solos aluviais & $\mathrm{V}$ FED & $\mathrm{FED}+\mathrm{C}$ \\
\hline Matias Cardoso & 18 & $14^{\circ} 59,12^{\prime}$ & $43^{\circ} 31,32^{\prime}$ & 85,83 & 425,5 & Bsh & 24 & 850 & Solos aluviais & V FED & FED \\
\hline Manga ${ }^{a}$ & 181 & $14^{\circ} 48,80^{\prime}$ & $43^{\circ} 55,89^{\prime}$ & 17,08 & 375,5 & Bsh & 24,4 & 828 & Solos aluviais & $\mathrm{V}$ FED & FED \\
\hline Manga $^{a}$ & 181 & $14^{\circ} 49^{\prime 2} 21,4^{\prime \prime}$ & $43^{\circ} 55^{\prime} 48,5^{\prime \prime}$ & 7,84 & 375,5 & Bsh & 24,4 & 828 & Solos aluviais & $\mathrm{V}$ FED & FED \\
\hline
\end{tabular}

Frag: Fragmento; GF: grupo fisionômico; VP: vegetação predominante; AI: área de influência; FES: Floresta Estacional Semidecidual; FED: Floresta Estacional Decidual; CSS: Cerrado Stricto Sensu; C: Cerrado. (a): mesmo fragmento, porém formado por áreas separadas; (*): base de classificação: mapa de solo (fonte: Geominas, 2003). 
Essa definição de grupos fisionômicos foi determinada para auxiliar nas análises comparativas das distribuições em ambientes mais homogêneos fisionomicamente, para, assim, ser possível analisar a real influência dentro e entre fisionomias recorrentes na área de estudo.

O método de amostragem utilizado foi o conglomerado em estágio único, com sistematização das unidades amostrais na área. Cada conglomerado foi composto por três subunidades de 10x25 m (250 $\mathrm{m}^{2}$ ), distantes 25 metros uma da outra. Ao longo do fragmento foram estabelecidos transectos distantes 100 metros entre conglomerados. Já ao longo de um transecto, os conglomerados ficaram a uma distância de 50 metros um do outro (Figura 2).

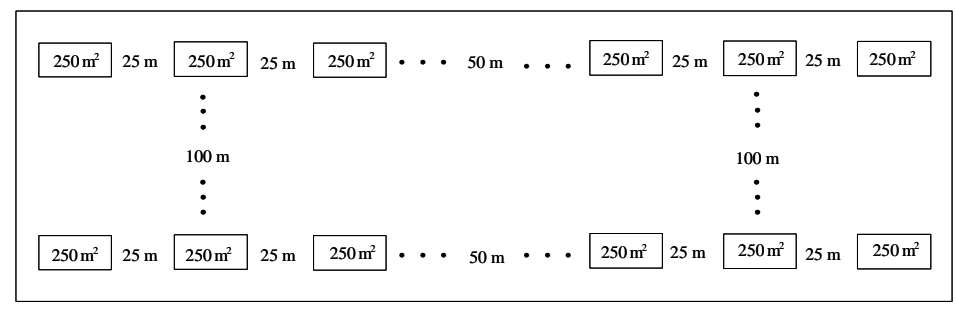

Figura 2. Representação da amostragem em conglomerado aplicada aos fragmentos da bacia do rio São Francisco (MG).

Figure 2. Cluster sampling scheme applied to each fragment from the São Francisco river watershed (MG).

A tabela 2 descreve a área dos fragmentos, o número de subunidades lançadas e a área amostral para o conjunto total, além de informar o somatório total dessas variáveis no estudo. As variáveis dendrométricas quantificadas nessas subunidades foram a altura total e a CAP (circunferência à altura do peito), para árvores que apresentavam a circunferência a 1,30 m de altura (CAP) igual ou superior a 15,7 cm.

Tabela 2. Informações do inventário florestal realizado em cada fragmento.

Table 2. Forest inventory information carried out for each fragment.

\begin{tabular}{lcccc}
\hline Fragmento & Município & Área (ha) & Área amostral (ha) & Número de subunidades \\
\hline 2 & Vargem Bonita & 21,83 & 0,600 & 24 \\
8 & Bambuí & 34,95 & 0,650 & 26 \\
100 & Iguatama & 5,17 & 0,450 & 18 \\
12 & Iguatama & 45,12 & 0,750 & 30 \\
14 & Iguatama & 43,40 & 1,050 & 42 \\
17 & Lagoa da Prata & 165,26 & 1,500 & 60 \\
22 & Abaeté & 2,88 & 0,375 & 15 \\
27 & Martinho Campos & 15,31 & 0,475 & 19 \\
117 & Lassance & 17,79 & 0,850 & 34 \\
70 & Lassance & 41,08 & 0,950 & 38 \\
116 & Jequitaí & 107,64 & 1,250 & 50 \\
105 & Ibiaí & 15,15 & 0,750 & 30 \\
110 & Ibiaí & 47,16 & 0,900 & 36 \\
112 & Santa Fé de Minas & 40,00 & 0,500 & 20 \\
114 & Brasilândia & 38,25 & 1,000 & 40 \\
66 & São Romão & 40,57 & 1,250 & 50 \\
55 & Urucuia & 9,34 & 0,625 & 25 \\
77 & Januária & 4,74 & 0,475 & 19 \\
181 & Manga & 24,93 & 1,050 & 42 \\
18 & Matias Cardoso & 85,83 & 0,850 & 34 \\
\hline Total & 20 & 806,40 & 16,30 & 652 \\
\hline
\end{tabular}

A identificação botânica das espécies arbóreo-arbustivas se deu a partir do reconhecimento de suas características dendrológicas no campo, com coleta do material botânico e posterior encaminhamento ao Herbário ESAL da Universidade Federal de Lavras, para concluir a identificação. A 
classificação dos indivíduos amostrados foi de família, gênero e espécie, tendo como referência Angiosperm Phylogeny Group (JUDD et al., 1999).

A suficiência amostral foi estimada e avaliada utilizando-se o procedimento da regressão linear com resposta em platô (REGRELRP), do Sistema para Análises Estatísticas SAEG V.5.0. No ajuste do modelo de platô, contou-se ainda com o fundamento da teoria do limite central, para garantir a confiabilidade no conjunto de dados selecionados para o ajuste. Segundo Ferreira (1988), o método de determinação de área mínima, conhecido como curva espécie-área, produz resultados subjetivos, ao contrário da regressão linear de platô.

Para verificar se existia semelhança entre distribuições diamétricas, bem como em altura, aplicou-se um teste estatístico não-paramétrico, denominado de Kolmogorov-Smirnov (SOKAL; ROHLF, 1995), em um nível de significância $(\alpha=0,05)$, pareando as amostras (ou fragmentos) duas a duas. Ao final, foram realizadas 190 combinações possíveis em cada distribuição, considerando os 20 fragmentos.

Considerando-se a distribuição diamétrica, a amplitude de classe foi de $5 \mathrm{~cm}$, e o valor inferior da primeira classe igual a $5 \mathrm{~cm}$. A análise dessa distribuição foi aplicada nos 20 fragmentos estudados, e o número de árvores da população foi extrapolado por hectare. Segundo Odum (1988), a sua caracterização permite inferir se as populações podem ser consideradas autoperpetuantes, ou seja, se apresentam indivíduos potenciais de migração, das menores classes para as maiores. Já para a distribuição hipsométrica, os indivíduos foram estratificados em classes com amplitude de 3 metros. Como na distribuição diamétrica, o número de árvores foi extrapolado por hectare.

O emprego dessa distribuição proporciona informações interessantes no sentido de se compreender o seu comportamento, que pode ser unimodal, multimodal e exponencial negativa ou decrescente (SOKAL; ROHLF, 1995). Autores como Scolforo (1998), Gomide (2004) e outros utilizaram esse mesmo teste em seus estudos, com o propósito de conhecer o comportamento da distribuição de frequência.

\section{RESULTADOS E DISCUSSÃO}

A aplicação da regressão linear de platô permitiu compreender o comportamento da amostragem ao longo de todos os levantamentos realizados. Verificou-se que a quantificação da variável espécie foi suficiente, ocorrendo assim a formação do platô nos 20 fragmentos inventariados. A tabela 3 mostra os parâmetros calculados e parte da Análise de Variância para os ajustes, bem como o número de dados pertencentes a cada equação (linear e platô) em cada fragmento.

A partir dos ajustes realizados, percebeu-se a ocorrência de uma amostragem ligeiramente superior à necessidade de cada local. Para se ter uma idéia desse valor, considere o número de conglomerados lançados a mais por fragmento igual a 4,1 em média, ou 12,3 subunidades, o que representou uma área média de $3.075 \mathrm{~m}^{2}$.

$\mathrm{Na}$ figura 3 são apresentados os gráficos da distribuição diamétrica dos indivíduos ao longo da bacia, apenas para os fragmentos 55 e 181, como exemplos. O comportamento dessa distribuição em todos os ambientes estudados foi parecido, ou seja, tendendo a exponencial negativa (ou "J invertido"). De acordo com Souza; Jesus (1994), a estrutura ecofisiológica da população pode ser percebida pelo comportamento da estrutura diamétrica de uma espécie ou grupo delas.

A população amostrada resultou em 16.235 indivíduos, com uma densidade de 1.015,89 indivíduos/ha e área basal média de $20,69 \mathrm{~m} / \mathrm{ha}$. A tabela 4 apresenta os dados quantitativos do inventário florestal realizado nas áreas.

A partir da análise dos dados, não foram detectadas lacunas nas classes inferiores de diâmetro, capaz de induzir uma redução no processo de suprimento ou recrutamento de indivíduos entre classes, para todos os fragmentos. Existe uma grande densidade de indivíduos nas menores classes de diâmetro, capaz de fornecer parte de seus representantes para as classes subsequentes, durante os períodos futuros, auxiliando na dinâmica e garantindo a continuidade arbórea da floresta.

Em todos os fragmentos, algumas classes mais elevadas apresentaram um desbalanceamento com relação às classes menores, como no caso do fragmento 77 , onde, na classe com valor central igual a $187,5 \mathrm{~cm}$, a densidade chegou a 2,11 indivíduos/ha, e nas 22 classes anteriores não foram constatados indivíduos, devido à presença de um indivíduo do gênero Cavanillesia (DAP $=193,28 \mathrm{~cm}$ ). Estudando um remanescente de Floresta Estacional Decidual de encosta (Goiás), Nascimento et al. (2004) encontrou um indivíduo pertencente ao mesmo gênero com DAP $=135,5 \mathrm{~cm}$. Segundo Martins (1996), as maiores classes de diâmetro apresentam um número reduzido de indivíduos, devido à grande mortalidade recorrente nas classes inferiores ao longo do tempo. 
Tabela 3. Parâmetros estimados dos modelos e parte da Análise de Variância utilizada para a seleção do modelo por fragmento.

Table 3. Estimated parameters and part of the Analysis of Variance used to select the model by fragment.

\begin{tabular}{|c|c|c|c|c|c|c|c|c|}
\hline \multirow{3}{*}{ Fragmento } & \multirow{2}{*}{\multicolumn{2}{|c|}{ Dados }} & \multicolumn{3}{|c|}{ Parâmetros } & \multirow{3}{*}{$\begin{array}{c}\text { SQD } \\
(\text { reta + platô })\end{array}$} & \multirow{3}{*}{$\mathbf{F}$} & \multirow{3}{*}{$\mathbf{R}^{2}$} \\
\hline & & & \multicolumn{2}{|c|}{ Reta } & \multirow{2}{*}{$\begin{array}{c}\text { Platô } \\
\mathbf{P}\end{array}$} & & & \\
\hline & Reta & Platô & $\beta_{0}$ & $\beta_{1}$ & & & & \\
\hline 2 & 17 & 7 & 32,4264 & 0,0267 & 149,1857 & 1156,0117 & 308,60 & 95,36 \\
\hline 8 & 18 & 8 & 20,6349 & 0,0183 & 104,9650 & 677,8314 & 323,23 & 95,28 \\
\hline 12 & 19 & 11 & 11,4774 & 0,0064 & 43,0091 & 142,6300 & 253,71 & 93,72 \\
\hline 14 & 25 & 17 & 12,5043 & 0,0037 & 36,1000 & 171,5435 & 216,72 & 90,41 \\
\hline 17 & 37 & 23 & 45,7024 & 0,0118 & 155,8696 & 3929,1429 & 424,12 & 92,37 \\
\hline 18 & 21 & 13 & 14,6705 & 0,0062 & 47,6954 & 252,8391 & 175,85 & 90,24 \\
\hline 22 & 11 & 4 & 19,2680 & 0,0167 & 66,8575 & 125,8332 & 168,30 & 94,92 \\
\hline 27 & 13 & 6 & 17,2865 & 0,0182 & 79,2333 & 273,3760 & 204,62 & 94,90 \\
\hline 55 & 18 & 7 & 11,1652 & 0,0087 & 51,8200 & 115,5222 & 433,58 & 96,44 \\
\hline 66 & 29 & 21 & 11,8755 & 0,0040 & 41,1586 & 283,8544 & 265,50 & 90,77 \\
\hline 70 & 24 & 14 & 30,6330 & 0,0123 & 106,4486 & 1109,0750 & 305,30 & 93,27 \\
\hline 77 & 13 & 6 & 10,0065 & 0,0115 & 48,4833 & 86,1479 & 307,36 & 96,55 \\
\hline 100 & 11 & 7 & 12,3764 & 0,0156 & 57,0057 & 166,5837 & 127,14 & 92,92 \\
\hline 105 & 13 & 17 & 11,4262 & 0,0087 & 61,5940 & 146,1816 & 144,45 & 92,92 \\
\hline 110 & 23 & 13 & 13,1841 & 0,0083 & 61,5940 & 345,7745 & 376,64 & 94,72 \\
\hline 112 & 13 & 7 & 6,0150 & 0,0049 & 22,5857 & 26,0734 & 150,72 & 93,20 \\
\hline 114 & 24 & 15 & 7,0041 & 0,0037 & 29,7427 & 102,5828 & 294,48 & 93,05 \\
\hline 116 & 28 & 22 & 24,9550 & 0,0091 & 90,0009 & 1135,7662 & 314,65 & 92,37 \\
\hline 117 & 19 & 15 & 8,377 & 0,0025 & 20,5787 & 40,2428 & 139,69 & 89,15 \\
\hline 181 & 21 & 21 & 9,4314 & 0,0044 & 32,9352 & 184,3187 & 164,95 & 89,67 \\
\hline
\end{tabular}

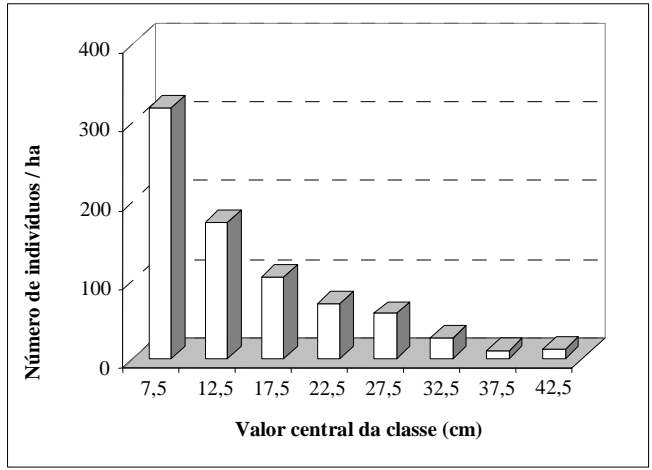

(55)

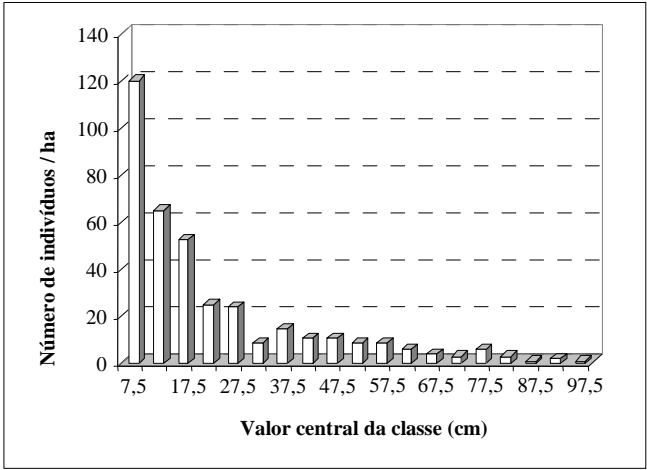

(181)

Figura 3. Distribuição diamétrica dos indivíduos nos fragmentos florestais.

Figure 3. Diametric distribution of the individuals in the forest fragments.

O fragmento 116 apresentou na primeira classe $67,3 \%$ dos indivíduos de toda a população, e os fragmentos 70 e 8 seguiram a mesma tendência, com a primeira classe diamétrica abrangendo $61,83 \%$ e $60,02 \%$ da população, respectivamente. Lopes et al. (2002) encontraram valores de 78,87\% da população nas duas primeiras classes de diâmetro no Parque Estadual do Rio Doce (MG). Machado et al. (2004) sugerem em seu trabalho que, quando a floresta apresentar um número excessivo de indivíduos nas menores classes de diâmetro, esse fato pode indicar uma perturbação na área. Além disso, fornece a ideia de sustentabilidade da floresta durante o processo natural de sucessão (CAVALCANTE, 1998; LOPES et al., 2002). 
Tabela 4. Comportamento da vegetação arbórea inventariada em cada fragmento, caracterizando a sua estrutura.

Table 4. Behavior of the arboreal vegetation inventoried in each fragment, characterizing its structure.

\begin{tabular}{|c|c|c|c|c|c|c|}
\hline Grupo fisionômico & Fragmento & N/ha & $\begin{array}{c}\text { d médio } \\
\text { (cm) }\end{array}$ & $\begin{array}{c}\text { d max } \\
\text { (cm) }\end{array}$ & $\begin{array}{c}\text { h med } \\
(\mathrm{m})\end{array}$ & $\begin{array}{c}\text { h max } \\
\text { (m) }\end{array}$ \\
\hline \multirow{9}{*}{ I } & 2 & 1585,32 & 11,89 & 67,48 & 10,05 & 24,80 \\
\hline & 8 & 1138,12 & 11,87 & 73,27 & 9,16 & 32,00 \\
\hline & 12 & 898,44 & 15,53 & 89,01 & 8,56 & 23,00 \\
\hline & 14 & 1294,85 & 12,45 & 82,76 & 8,62 & 21,00 \\
\hline & 17 & 1464,07 & 11,16 & 93,90 & 9,45 & 21,00 \\
\hline & 22 & 1357,50 & 11,47 & 76,38 & 9,03 & 22,00 \\
\hline & 27 & 1265,11 & 12,03 & 82,76 & 9,75 & 27,60 \\
\hline & 70 & 1498,05 & 10,60 & 69,74 & 6,57 & 17,00 \\
\hline & 100 & 1031,01 & 14,38 & 90,63 & 8,45 & 20,00 \\
\hline Média I & & 1281,38 & 12,38 & 80,66 & 8,85 & 23,16 \\
\hline \multirow{3}{*}{ II } & 112 & 730,00 & 12,47 & 90,08 & 8,49 & 22,60 \\
\hline & 114 & 494,00 & 14,32 & 84,35 & 8,86 & 26,00 \\
\hline & 117 & 901,99 & 13,39 & 95,53 & 9,26 & 23,60 \\
\hline \multirow[t]{2}{*}{ Média II } & & 708,66 & 13,39 & 89,99 & 8,87 & 24,07 \\
\hline & 66 & 509,60 & 18,59 & 135,92 & 10,11 & 23,00 \\
\hline \multirow{3}{*}{ III } & 105 & 1133,16 & 11,43 & 57,30 & 7,18 & 15,80 \\
\hline & 110 & 727,05 & 12,93 & 76,94 & 8,11 & 22,00 \\
\hline & 116 & 1132,80 & 10,35 & 56,66 & 7,36 & 25,00 \\
\hline Média III & & 875,65 & 13,32 & 81,70 & 8,19 & 21,45 \\
\hline IV & 55 & 779,20 & 14,57 & 46,59 & 8,04 & 16,00 \\
\hline Média IV & & 779,20 & 14,57 & 46,59 & 8,04 & 16,00 \\
\hline \multirow{3}{*}{$\mathrm{V}$} & 18 & 793,80 & 14,87 & 139,23 & 8,27 & 28,00 \\
\hline & 77 & 1212,48 & 12,06 & 193,28 & 8,21 & 21,00 \\
\hline & 181 & 371,28 & 22,25 & 97,40 & 9,45 & 25,00 \\
\hline Média V & & 792,52 & 16,39 & 143,30 & 8,64 & 24,67 \\
\hline Média geral & & 1015,89 & 13,43 & 89,96 & 8,65 & 22,82 \\
\hline
\end{tabular}

Silva Júnior (2004), estudando uma mata de galeria em Brasília, constatou que 52\% das árvores pertenciam à classe de 5-10 cm, e $91 \%$ apresentaram um DAP $<30 \mathrm{~cm}$. Em outra área ciliar, Garcia et al. (2003) constataram que a distribuição diamétrica seguiu um comportamento em $\mathrm{J}$ invertido, com a maioria dos indivíduos $(62 \%)$ concentrada na primeira classe $(5-10 \mathrm{~cm})$.

Por outro lado, os fragmentos 181, 66 e 55 resultaram nas menores porcentagens de indivíduos na primeira classe diamétrica, com $32,32 \%, 32,81 \%$ e $41,07 \%$ da população, respectivamente. Aparício (2006), estudando um fragmento de Mata Atlântica, encontrou na primeira classe o valor de 39,56\% (DAP até $9,77 \mathrm{~cm}$ ).

A floresta presente no fragmento 55 proporcionou um ambiente onde as espécies inventariadas desenvolvem um porte diamétrico baixo. O maior DAP encontrado foi de $46,59 \mathrm{~cm}$, valor muito pequeno se comparado com a média da bacia, que foi de $89,96 \mathrm{~cm}$.

A figura 4 mostra a distribuição das alturas dos fragmentos (55 e 8) em classes com a amplitude de 3 metros. O número de classes variou de 6 nos fragmentos 55, 70 e 105 a 11 no fragmento 8 . Lopes et al. (2002), em estudo no Parque Estadual do Rio Doce (MG), constataram que a distribuição hipsométrica abrangeu um total de 11 classes de altura, com 2,5 m de amplitude, e que somente nas classes de 6-12 m de altura os indivíduos representaram um total de $65 \%$.

Todos os fragmentos apresentaram indivíduos nas seis primeiras classes de altura. A classe mais representativa e que abrangeu o maior número de indivíduos foi a de 6 a 9 m $(43,37 \%)$, demonstrando que o comportamento foi semelhante a este nível do dossel. 


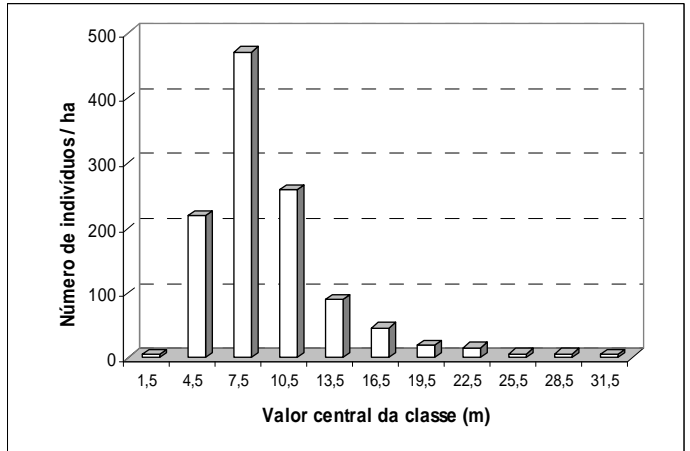

(8)

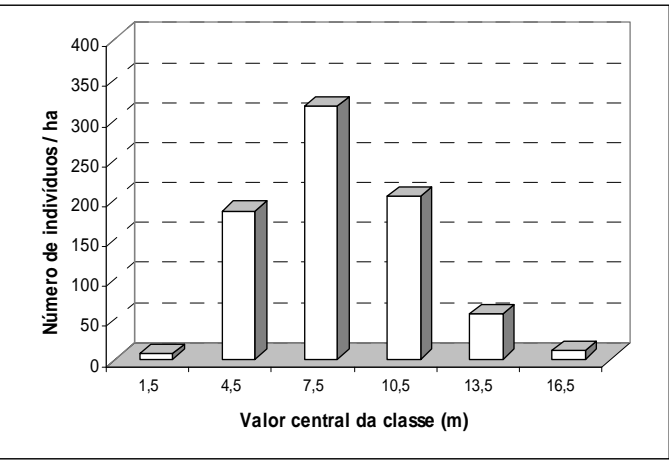

(55)

Figura 4. Distribuição das alturas dos indivíduos amostrados nos fragmentos florestais. Figure 4. Height distribution of the individuals in the forest fragments.

A figura 5 mostra que a altura média dos fragmentos oscilou de $6,57 \mathrm{~m}$, no fragmento 70 , a 10,11 m no fragmento 66. Os fragmentos 66 e 2 não apresentaram as maiores alturas de indivíduos, mas, em média, estas foram as florestas com o dossel mais elevado.

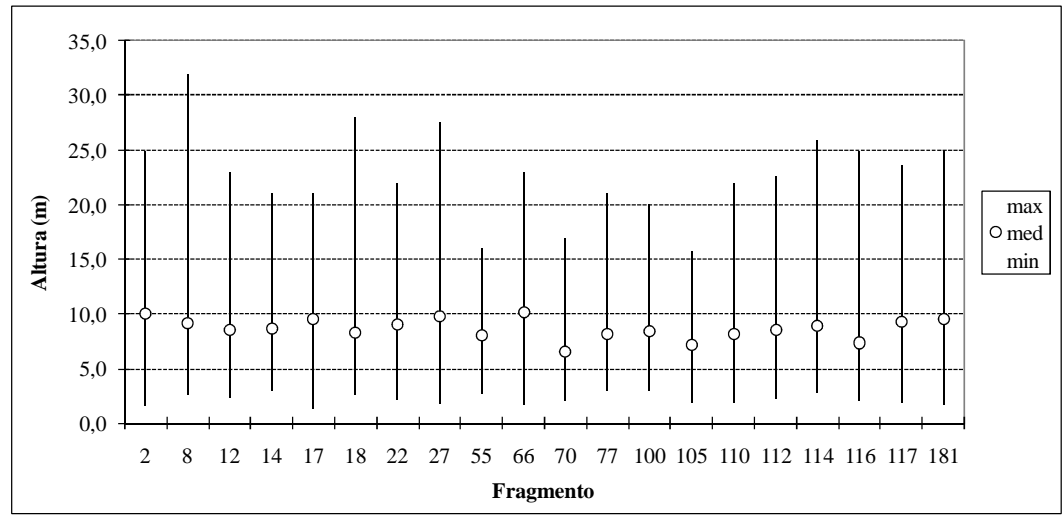

Figura 5. Representação gráfica da altura das vegetações $(\max =$ máxima, med $=$ média $\mathrm{e}$ min $=$ mínima).

Figure 5. Graphic representation of the vegetation height $(\max =\operatorname{maximum}, \operatorname{med}=\operatorname{medium}$ and $\min =$ minimum height).

A figura 6, letras (a) e (b), retrata padrões de distribuição em altura observada que refletem, respectivamente, a maior discrepância e a maior semelhança entre os pares de fragmentos estudados, detectados por meio do teste de Kolmogorov-Smirnov. Os fragmentos 12 e 181 (Figura 6a) tiveram a maior discrepância na frequência de altura, ou seja, dos 190 conjuntos formados pelos 20 fragmentos pareados 2 a 2, aqueles fragmentos formam o par mais diferente de padrões de altura. Por outro lado, os conjuntos 12 e 112 (Figura 6b) demonstram características opostas ao do conjunto anterior, desenvolvendo praticamente a mesma distribuição em altura.

A distribuição diamétrica mostrada na figura 6 pelas letras (c) e (d), a partir do teste de Kolmogorov-Smirnov, mostra que os fragmentos 70 e 181 apresentam as maiores diferenças entre todos os pares de áreas estudadas, indicando que realmente esses locais não se correlacionam em termos de distribuição diamétrica. O oposto ocorre com o fragmento 100, caracterizado pela Floresta Estacional Semidecidual, e o fragmento 18, formado pela Floresta Estacional Decidual, para os quais as curvas de distribuição apresentam-se praticamente aderidas uma à outra.

Analisando a bacia como um todo, percebeu-se que a classificação da vegetação pouco influi no padrão de distribuição das curvas de frequência do diâmetro e da altura (Tabela 5). Dessa forma, dos 190 
pares de comparação estabelecidos, apenas 17 (F2 x F22, F8 x F27, F8 x F112, F8 x F117, F12 x F100, F12 x F114, F12 x F18, F14 x F77, F17 x F22, F100 x F55, F112 x F110, F55 x F18, F110 x F18, F12 x F55, F100 x F114, F14 x F22 e F114 x F110), ou 8,94\%, apresentavam uma igualdade, tanto na distribuição diamétrica como na distribuição em altura. Isso demonstra uma semelhança estrutural nessas variáveis dendrométricas.

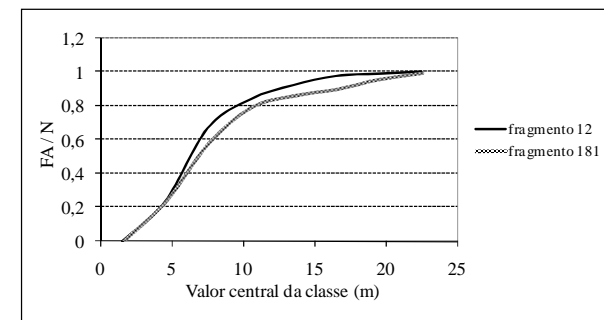

(a)

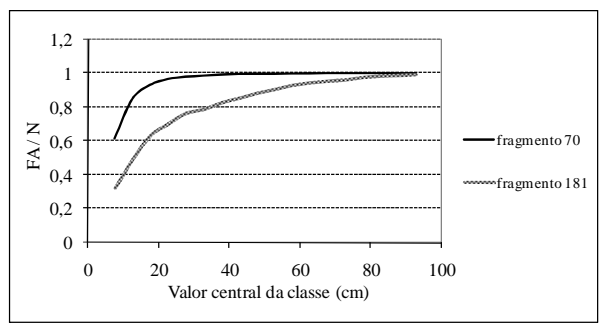

(c)

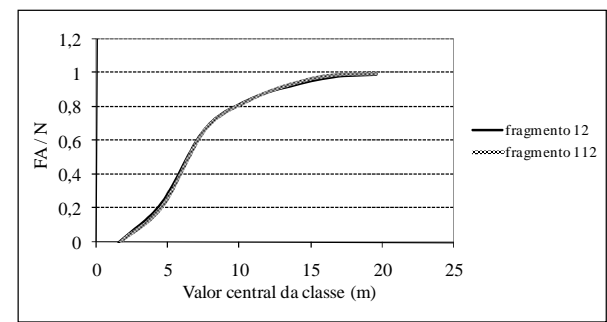

(b)

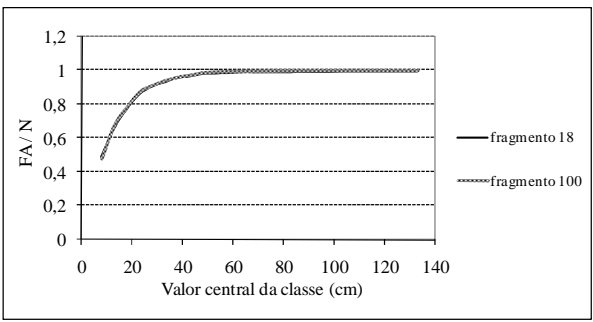

(d)

Figura 6. Análise comparativa das distribuições diamétricas e em altura entre fragmentos, a partir do teste de Kolmogorov-Smirnov ( ${ }^{\alpha}=0,05$ ), em que (a) e (b) representam as alturas, e (c) e (d) os diâmetros.

Figure 6. Comparative analysis of the diametric and hypsometric distributions between fragments using the Kolmogorov-Smirnov test $(\alpha=0,05)$, where (a) and (b) represent heights, and (c) and (d) diameters.

Na tabela 6, os valores 1 e 2 representam a igualdade na distribuição em altura e igualdade na distribuição diamétrica, respectivamente. Aproximadamente 12,11\% dos pares de fragmentos apresentam a mesma distribuição de frequência em altura, e $25,26 \%$ a mesma distribuição de frequência em diâmetro. Isso indica que, nos ambientes inventariados, o que predomina é a igualdade de frequência diamétrica entre fragmentos, e não a igualdade de frequência em altura. Uma possível explicação para esse fato é que a altura esteja mais correlacionada com os fatores ambientes inventariados de cada local (qualidade de sítio), quando comparado com a distribuição diamétrica.

O nível $O$ foi o mais recorrente, sendo detectado em 102 pares de fragmentos (ou 53,68\% dos pares estudados). Ele sugere a ideia de total disparidade das distribuições estudadas entre os pares de fragmentos comparados. A grande representatividade do nível $O$ caracteriza, na sua maioria, uma complexidade de ambientes estruturalmente heterogêneos nas áreas de preservação permanente, associados aos cursos d'água ao longo da bacia do rio São Francisco. Isso indica que, realmente, um dos inúmeros fatores que determinam o desenvolvimento de uma floresta, tanto em dossel como em diâmetro, é a interação entre a vegetação e o ambiente.

O fragmento 66 foi o que mais se destacou no nível 0 , com $94,73 \%$ de ocorrência, indicando que suas distribuições são raras na bacia. Por outro lado, o fragmento 112 foi o que apresentou o menor número de desigualdades de diâmetro e altura (nível 0 ), possibilitando uma alta frequência nos outros níveis.

Já o fragmento 70 apresentou apenas correlação em diâmetro, e o oposto foi observado no fragmento 181, onde somente correlações em frequência de altura foram observadas. No nível 3, os fragmentos mais recorrentes foram o 8 e o 12 , pareando 4 vezes com outros fragmentos. Já os menos recorrentes foram $2,66,70,105,116$ e 181, que não estabeleceram nenhuma relação no terceiro nível. 
Tabela 5. Análise da distribuição de diâmetro (a) e altura (b) entre os fragmentos, pelo teste de Kolmogorov-Smirnov ( $\alpha=0,05)$.

Table 5. Analysis of the diametric (a) and hypsometric (b) distributions among the fragments, by the Kolmogorov-Smirnov test $(\alpha=0,05)$.
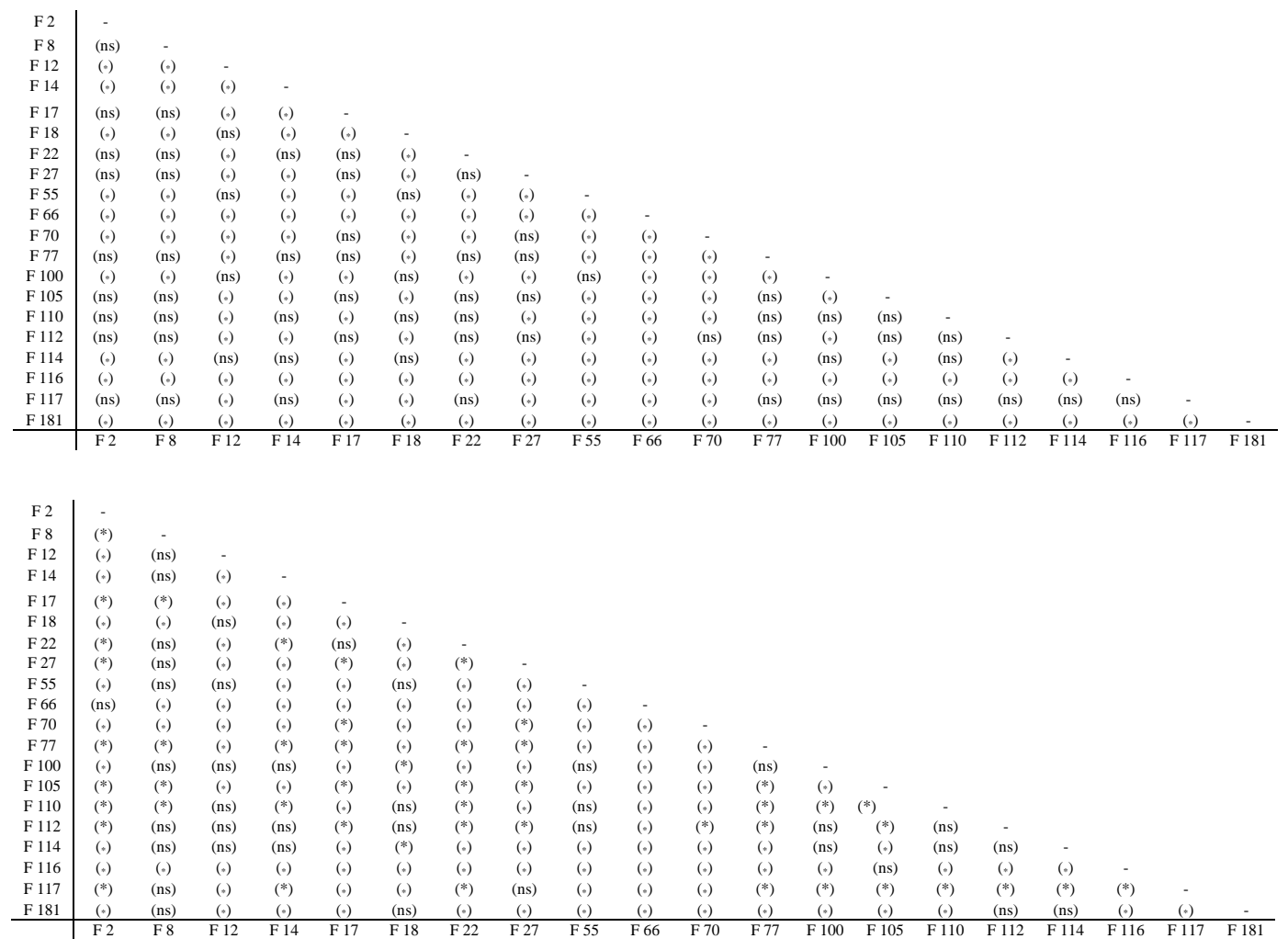

$(*)=$ significativo; $(\mathrm{ns})=$ não significativo a $(\alpha=0,05)$.

Tabela 6. Comportamento da distribuição diamétrica e em altura a partir do teste de KolmogorovSmirnov $(\alpha=0,05)$.

Table 6. Diameter and height distributions behaviors using the Kolmogorov-Smirnov test ( $\left.{ }^{\alpha}=0,05\right)$.

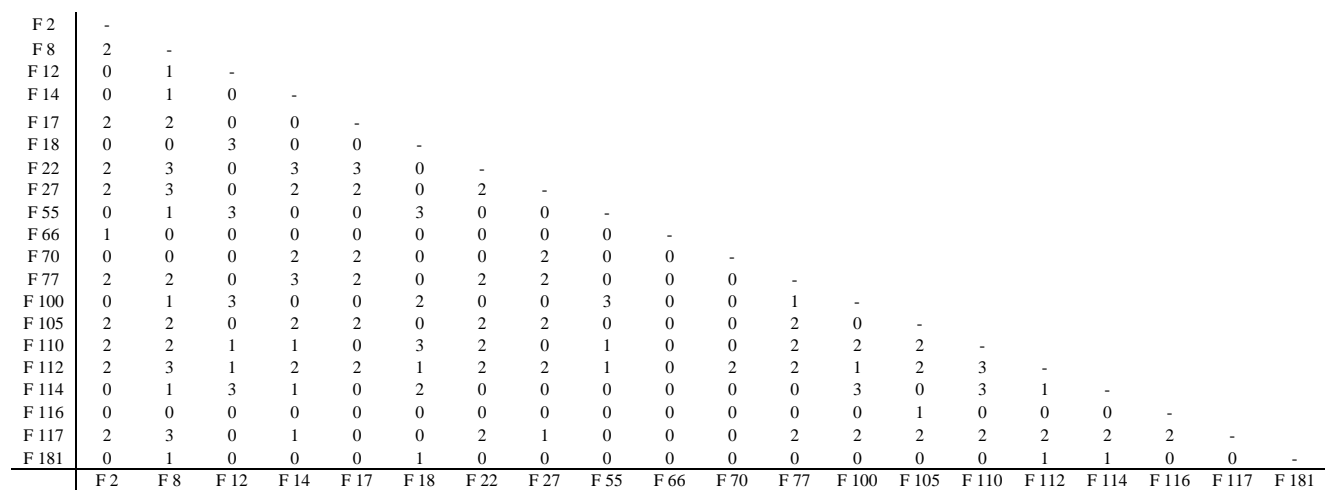

$0,1,2$ e 3 = níveis de comportamento das distribuições: 0 - indica que as distribuições em diâmetro e em altura foram diferentes entre os fragmentos pareados; 1 - apenas a distribuição em altura foi semelhante no par; 2 - apenas a distribuição diamétrica foi semelhante no par; 3 - as distribuições em diâmetro e em altura foram semelhantes no par.

A formação dos grupos fisionômicos próximos à Bacia permitiu distinguir melhor e com maior 
detalhamento as vegetações florestais inseridas na paisagem, distinguindo áreas mais homogêneas floristicamente e permitindo comparações entre áreas com o mesmo padrão vegetacional. Assim, aplicando o teste de Kolmogorov-Smirnov a 5\% de probabilidade, percebeu-se que o comportamento da distribuição diamétrica nem sempre seguia um comportamento semelhante entre os fragmentos pertencentes ao mesmo grupo fisionômico, o mesmo ocorrendo para a distribuição hipsométrica. Esse fato mostrou que o agrupamento de áreas floristicamente semelhantes ainda não garante uma total igualdade desses padrões de distribuição (hipsométrica e diamétrica). Uma hipótese a respeito dessa variação pode ser atribuída à fragilidade ambiental de cada local, uma vez que as distribuições diamétrica e hipsométrica são altamente dependentes do grau de conservação do hábitat.

O comportamento das distribuições em cada grupo fisionômico encontra-se na tabela 7 , na qual os valores $0,1,2$ e 3 seguem a mesma lógica descrita anteriormente. A partir dos resultados, percebeu-se que o Grupo Fisionômico I apresentou o seguinte comportamento entre os pares de fragmentos: (3) 13,89\%, (2) 44,44\%, (1) 5,56\% e (0) 36,11 \%; Grupo Fisionômico II: (3) 0\%, (2) 66,67\%, (1) 33,33 \% e (0) 0 \%; Grupo Fisionômico III: (3) 0\%, (2) 16,67\%, (1) 16,67\% e (0) 66,66\%; Grupo Fisionômico IV apresenta apenas 1 fragmento, logo não há comparações; Grupo Fisionômico V: (3) 0\%, (2) 0\%, (1) $33,34 \%$ e $(0) 66,66 \%$.

Tabela 7. Comportamento das distribuições diamétrica e hipsométrica para os grupos fisionômicos, a partir do teste de Kolmogorov-Smirnov ( $\alpha=0,05$ ).

Table 7. Diametric and hypsometric distribution behavior for the physiognomic groups using the Kolmogorov-Smirnov test $(\alpha=0,05)$.
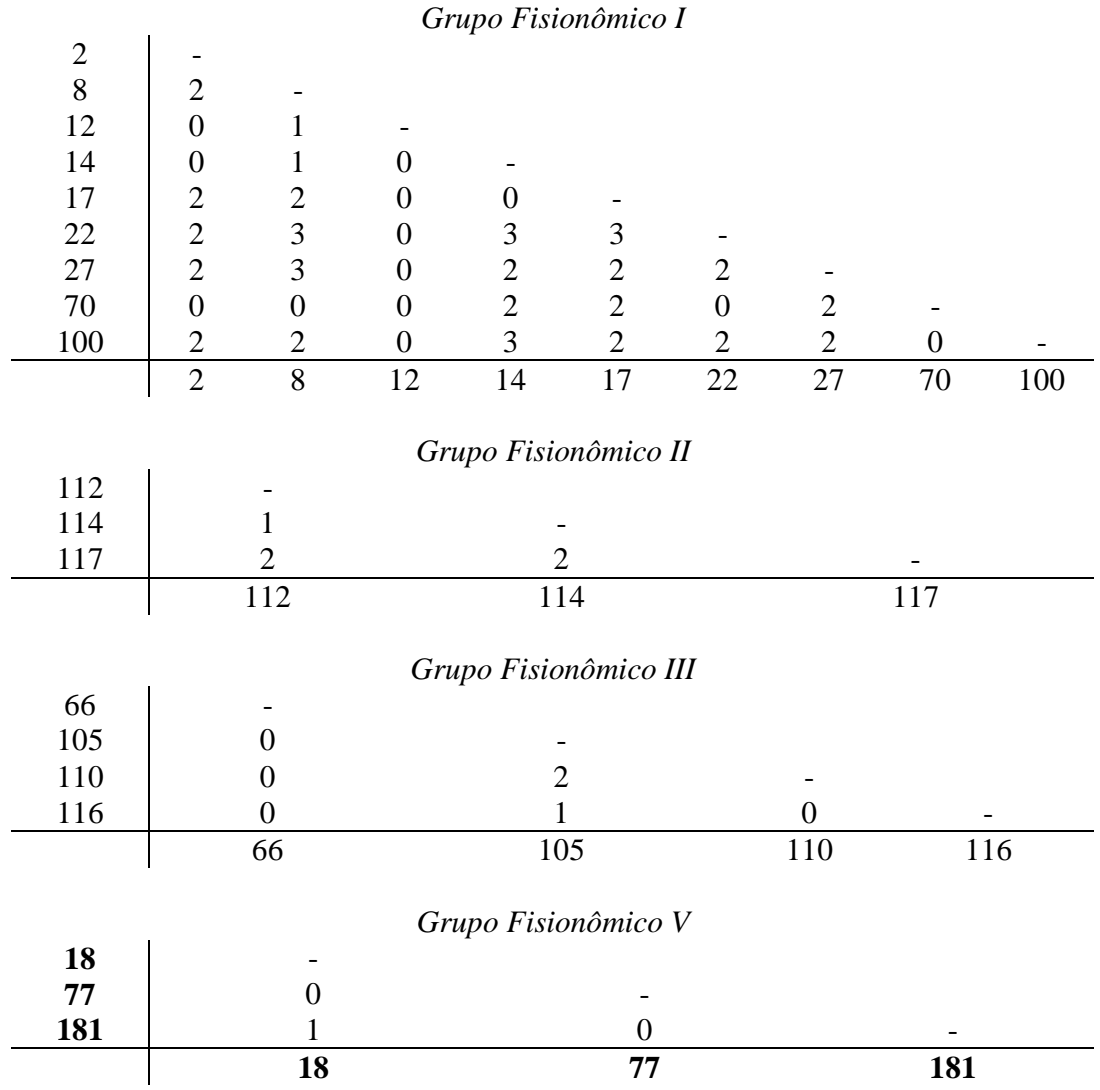

Dentro do grupos fisionômicos I e II, a semelhança da distribuição diamétrica entre os pares foi a que mais ocorreu, $44,44 \%$ e $66,67 \%$, respectivamente. Já para os grupos III e V, a completa diferença entre cada distribuição foi a mais recorrente (66,66\% para ambos). Porém apenas o grupo fisionômico I foi o que apresentou pares de fragmentos com igualdade tanto na distribuição diamétrica como na hipsométrica. Esse fato pode ser atribuído a um maior grau de "pureza" desse grupo, uma vez que, pela 
classificação (Tabela 1), não há traços de influência de outras fisionomias, ou a um pequeno grau se comparado com os outros grupos. Entretanto, ao se analisarem os outros grupos fisionômicos, estes estão inseridos em áreas ecotonais, ou seja ambientes influenciados por distintas fisionomias, o que pode ser uma das possíveis explicações para o fato.

Em áreas de influências ou transição entre fisionomias na Bacia, a altura e o diâmetro apresentaram o mesmo comportamento entre os pares de fragmentos no grupo fisionômico III. Já no grupo fisionômico $\mathrm{V}$, apenas a semelhança da distribuição hipsométrica foi constatada.

No grupo Fisionômico V, os fragmentos 18 e 181 são classificados como Floresta Estacional Decidual e não sofrem influências de outras fisionomias (Tabela 1). Dessa forma, apenas a altura entre esses fragmentos foi a que apresentou o mesmo comportamento pelo teste de Kolmogorov-Smirnov a 5\% de probabilidade. $\mathrm{O}$ fragmento 77 também pertencente a esse grupo, não apresentou qualquer semelhança na distribuição diamétrica e hipsométrica com os outros membros do grupo. Se observada a sua formação florestal, percebe-se que há certa influência de outra fisionomia.

\section{CONCLUSÕES}

$\mathrm{O}$ teste de Kolmogorov-Smirnov $(\alpha=0,05)$ não detectou diferenças significativas nos fragmentos que pertenciam a grupos fisionômicos distintos $\left(\mathrm{F}_{8} \times \mathrm{F}_{112}, \mathrm{~F}_{8} \times \mathrm{F}_{117}, \mathrm{~F}_{12} \times \mathrm{F}_{114}, \mathrm{~F}_{12} \times \mathrm{F}_{18}, \mathrm{~F}_{14} \times\right.$ $\mathrm{F}_{77}, \mathrm{~F}_{100} \times \mathrm{F}_{55}, \mathrm{~F}_{112} \times \mathrm{F}_{110}, \mathrm{~F}_{55} \times \mathrm{F}_{18}, \mathrm{~F}_{110} \times \mathrm{F}_{18}, \mathrm{~F}_{12} \times \mathrm{F}_{55}, \mathrm{~F}_{100} \times \mathrm{F}_{114}, \mathrm{~F}_{14} \times \mathrm{F}_{22}$ e $\mathrm{F}_{114} \times \mathrm{F}_{110}$ ), o que permite concluir que não existe um padrão característico de distribuição de frequência (diâmetro e altura) para cada um dos 5 grupos fisionômicos pertencentes à Bacia.

Em áreas consideradas ecótonos, dentro dos grupos fisionômicos, não há preferência na semelhança da distribuição diamétrica ou hipsométrica, indicando que essas variáveis dependem exclusivamente das interações entre fisionomias.

Os grupos mais homogêneos floristicamente apresentaram maior equilíbrio no padrão da distribuição diamétrica e hipsométrica, frente a ambientes mais heterogêneos.

\section{REFERENCIAS}

APARÍCIO, P. S.; COSTA JUNIOR, R. F.; SILVA, W. C.; OLIVEIRA, C. A. N.; FERREIRA, R. L. C.; MARANGON, L. C. Distribuição diamétrica e hipsométrica de um fragmento de mata atlântica no município de Catende, Pernambuco. In: SIMPÓSIO DE PÓS-GRADUAÇÃO EM CIÊNCIAS FLORESTAIS, 2. 2006, Piracicaba. Anais....Piracicaba: ESALQ-USP, 2006.1 CD-ROM.

CAVALCANTE, D. Florística de um remanescente florestal transacional no município de Guaratinguetá, SP. 103 p. Dissertação (Mestrado) - Universidade de São Paulo, Rio Claro, 1998.

DISLICH, R.; CERSÓSIMO, L.; MANTOVANI, W. Análise da estrutura de fragmentos florestais no Planalto Paulistano, SP. Revista Brasileira de Botânica, São Paulo, v. 24, n. 3, p. 321-332, 2001.

EMPRESA DE PESQUISA AGROPECUÁRIA DE MINAS GERAIS. Levantamento de reconhecimento detalhado dos solos da área sob influência do reservatório de Três Marias, MG. Belo Horizonte: SNL, 1978. 263 p. (Boletim Técnico do SNL, S57).

FELFILI, J. M.; SILVA JUNIOR, M. C. A comparative study of cerrado (stricto sensu) vegetation in central Brazil. Journal of Tropical Ecology, Cambridge, v. 9, n. 3, p. 227-289, 1993.

FERREIRA, R. L. C. Análise estrutural da vegetação da Estação Florestal de Experimentação de Açu, RN, como subsídio básico para o manejo florestal. 91 p. Dissertação (Mestrado em Ciências Florestais) - Universidade Federalde Viçosa, Viçosa, MG, 1988.

GARCIA, C. C.; ARAUJO, C. G.; BIANCHINI, E. Distribuição diamétrica e de altura de uma comunidade arbórea ciliar na bacia do rio Tibagi, norte do Paraná. In: CONGRESSO DE ECOLOGIA DO BRASIL, 6., 2003, Fortaleza. Anais de trabalhos completos... Fortaleza: Editora da Universidade Federal do Ceará, 2003. p. 427-428.

GEOMINAS. Disponível em: <http://www.geominas.mg.gov.br>. Acesso em: 14/08/2003. 
GOMIDE, L. R. Um modelo fitogeográfico para a bacia do rio São Francisco, em Minas Gerais. 268 p. Dissertação (Mestrado em Engenharia Florestal) - Universidade Federal de Lavras, Lavras, 2004 . .

HUBBELL, S. P.; FOSTER, R. B. La estructura espacial em gran escala de um bosque neotropical. Revista de Biologia Trapical, São José, v. 35, n. 1, p. 7-22, 1987.

INSTITUTO BRASILEIRO DE GEOGRAFIA E ESTATÍSTICA. Manual técnico da vegetação brasileira. Rio de Janeiro, 1992. $92 \mathrm{p}$.

JUDD, W. S.; CAMPBELl, C. S.; KELlOG, E. A.; STEAVENS, P. F. Plant systematics: a phylogenetic approach. Massachusetts: Sinauer Associates, 1999. 620 p.

LOETSCH, F.; ZÖHRER, F.; HALLER, K. E. Forest inventory. Munich: BLV, 1973. v. 2.

LOPES, W. P.; SILVA, A. F.; NETO, J. A. A. M. Estrutura fitossociológica de um trecho de vegetação arbórea no Parque Estadual do Rio Doce - Minas Gerais, Brasil. Acta botânica brasílica, Porto Alegre, v. 16, n. 4 , p. $443-456,2002$.

MACHADO, E. L. M.; OLIVEIRA-FILHO, A. T.; CARVALHO, W. A. C.; SOUZA, J. S.; BORÉM, R. A. T.; BOTEZELLI, L. Análise comparativa da estrutura e flora do compartimento arbóreo-arbustivo de um remanescente florestal na fazenda Beira Lago, Lavras, MG. Revista Árvore, Viçosa, MG, v. 28, n. 4, p. 499-516, 2004.

MARTINS, F. R. Aspectos da organização de uma comunidade arbórea florestal: um estudo prático. [S.1.: s.n.], 1996. p. 18. Apostila do curso de ecologia vegetal, BT-682.

NIMER, E. Climatologia do Brasil. Rio de Janeiro: IBGE, 1979. 422 p.

ODUM, E. P. Ecologia. Rio de Janeiro: Guanabara Koogan, 1988. 434 p.

PIRES O'BRIEN, M. J.; O'BRIEN, C. M. Ecologia e modelamento de Florestas Tropicais. Belém: FCAP, 1995. $400 \mathrm{p}$.

PULZ, F. A.; SCOLFORO, J. R.; OLIVEIRA, A. D.; MELLO, J. M.; OLIVEIRA-FILHO, A. T. Acuracidade da predição da distribuição diamétrica de uma floresta inequiânea com a matriz de transição. Cerne, Lavras, v. 5. p. 1-14, 1999.

RODRIGUES, R. R.; GANDOLFI, S. Restauração de florestas tropicais: subsídios para uma definição metodológica e indicadores de avaliação e monitoramento. In: DIAS, L. E.; MELLO, J. W. V. (Ed.). Recuperação de áreas degradadas. Viçosa, MG: UFV, 1998. p. 203-215.

SAEG - Sistema para Análise Estatística e Genética (SAEG v. 5.0): manual de uso. Viçosa: UFVFunarbe, 1997.

SANO, S. M.; ALMEIDA, S. P. Cerrado: ambiente e flora. Planaltina: EMBRAPA CPAC, 1998. 556 p.

SCOLFORO, J. R. S. Modelagem do crescimento e da produção de florestas plantadas e nativas. Lavras: UFLA/FAEPE, 1998. 463 p.

SILVA, A. L.; SCARIOT, A. Composição florística e estrutura da comunidade arbórea em uma Floresta Estacional Decidual em afloramento calcário (Fazenda São José, São Domingos, GO, Bacia do rio Paranã). Acta botânica brasílica, Porto Alegre, v. 17, n. 2, p. 305-313, 2003.

SILVA JÚNIOR, M. C. Fitossociologia e estrutura diamétrica da mata de galeria do taquara, na reserva ecológica do IBGE, DF. Revista Árvore, Viçosa, MG, v. 28. n. 3. p. 419-428, 2004.

SOKAL, R. R.; ROHLF, F. J. Biometry: the principles and practice of statistics in biological research. 3. ed. New York: W. H. Freeman, 1995. 887 p.

SOUZA, A. L.; JESUS, R. M. Distribuição diamétrica de espécies arbóreas da Floresta Atlântica: análise de agrupamento. Viçosa, MG: Sociedade de Investigações Florestais, 1994. 30p. (Boletim Técnico, 10). 